\title{
The upgrade programme of the CMS Tracker at SLHC
}

\section{G. Hall ${ }^{1}$ for the CMS collaboration}

Blackett Laboratory, Imperial College

London SW7 2AZ, UK

E-mail: g.halleimperial.ac.uk

The CMS experiment at LHC is planning a major upgrade of its tracking system to adapt to expected increases in luminosity of the accelerator. Present ideas suggest it will be achieved in two stages, with a major long shutdown after the experiments have reached their design lifetimes, about ten years after start-up. At this time the accelerator will increase the luminosity to $10^{35} \mathrm{~cm}^{-2} \cdot \mathrm{s}^{-1}$. The new tracker will then have to cope with several hundred interactions per bunch crossing and fluxes of thousands of charged particles emerging from the $40 \mathrm{MHz}$ collisions.

This will require major developments of detector technology and R\&D has begun to address the expected challenges. Among the most important are the radiation tolerance of sensors and other components, the provision and distribution of power for both electronics and sensors, the removal of heat loads, which may significantly exceed those in the present detector, and the development of low power, but highly performing electronics in more advanced technologies.

CMS has also identified another novel requirement, which is to utilise tracker data in the first level trigger, which must maintain the $100 \mathrm{kHz}$ rate for compatibility with existing sub-detector systems while increasing the decision latency by only a few $\mu \mathrm{s}$.

The motivations for the upgrade and recent progress in several aspects of the R\&D are described.

17th International Workshop on Vertex detectors

Utö Island, Sweden

July $28^{\text {th }}-1^{\text {st }}$ August, 2008

Speaker 


\section{Introduction}

The CMS experiment at the CERN Large Hadron Collider was designed nominally for operation for 10 years. The maximum peak luminosity of $10^{34} \mathrm{~cm}^{-2} \mathrm{~s}^{-1}$ is expected to be reached gradually after a few years, eventually achieving an integrated luminosity of about $50 \mathrm{fb}^{-1} /$ year, taking into account operational efficiency. Given the various uncertainties in the details of machine operation and experiment performance it is too soon to say whether the nominal detector lifetime might be exceeded. Certainly only when the LHC and experiments have been running for some time will it be possible to understand whether safety factors included at the design stage will permit operation beyond the original ten year goal. What is not in doubt that such factors might gain a few more years of good quality data taking at full luminosity but not more.

The upgrade of the LHC, to become Super-LHC (SLHC), which has been discussed for some years [1] foresees improvements to operate the accelerator at an order of magnitude higher luminosity of $10^{35} \mathrm{~cm}^{-2} \mathrm{~s}^{-1}$. Under these conditions, it is believed that most of CMS will survive the predicted radiation doses and inevitable ageing and continue to perform well with few changes. It is expected that over the coming years there can be modest continuous improvements to some accessible features of the experiment but eventually the trigger electronics and data acquisition systems would take maximum advantage of evolution of electronics and computing technologies to undergo substantial upgrades. This would contribute to handling the large data volumes and high rates anticipated at SLHC.

Most sub-detector systems in CMS will not be substantially changed, except for some accessible electronics. It is therefore important to maintain compatibility with existing systems and, since much of CMS is highly inaccessible, it is essential to try to retain the present Level 1 trigger rate limit of $100 \mathrm{kHz}$. The trigger decision latency could increase from the present value of about $3.2 \mu \mathrm{s}$ to a maximum of $6.4 \mu \mathrm{s}$, limited by digital pipeline lengths in the electromagnetic calorimeter.

The notable exception is the tracking system, whose performance will eventually be degraded by radiation damage caused by the immense particle fluxes to which it will be exposed. Greater radiation tolerance will be required for SLHC operation, especially for the sensors, and R\&D has been under way for some years [2] studying the most promising innovations. In contrast, it seems ASIC electronics could easily withstand SLHC radiation levels but the $0.25 \mu \mathrm{m}$ CMOS technology, pioneered by CMS and used for much of its electronics will probably be superseded by more advanced, finer feature size processes.

However, in the congested SLHC environment of 300-400 events per beam crossing, producing thousands of particles emerging from the interaction region, higher detector granularity is required to obtain physics performance matching what is currently achieved. CMS has also identified another major new challenge, which is to use tracker data to contribute to the first level hardware trigger decision. Both of these have important implications for a new system, including for the number of detector channels and the power required as well as other significant technical difficulties to be overcome. Hence a vigorous $R \& D$ effort is already needed. 


\section{Physics Requirements}

The main motivation for a luminosity upgrade is to deliver more statistics to improve physics studies beyond those possible at LHC. To benefit, it is essential that detector performance remains similar to that at LHC, while higher luminosity implies higher particle fluxes, detector occupancies, trigger rates, and radiation damage. Thus, the remarkable achievements in detector development for LHC must be surpassed in future in this even more challenging environment.

Until LHC has operated for some years, the principal future physics areas will remain speculative. However, there are many examples of the SLHC physics potential $[1,3]$, such as improvements in determination of Standard Model parameters, e.g. triple Higgs couplings or triple and quartic gauge boson couplings, and studies of new physics hopefully awaiting discovery at the LHC, such as determination of SUSY parameters and sparticle spectroscopy. These efforts will be accompanied by searches to higher masses for composite quarks, new heavy gauge bosons, multi-TeV squarks and gluinos, and extra dimensions. Investigations of rare processes such as FCNC top decays, Higgs-pair production, or multi gauge boson production will be carried out.

The challenges become evident when one reflects on statistics which might be accumulated. Studies for the CMS Physics Technical Design Report [4] demonstrate, for example, that a successful search for the simplest Standard Model Higgs might produce from a few hundred to a few thousand events, depending on mass, from an integrated luminosity of 300 $\mathrm{fb}^{-1}$, corresponding to about ten years of LHC operation.

Whatever Higgs variant is discovered, more information on its properties will be needed and subtle investigations to study couplings are needed. Simulations of $\mathrm{HH}$ production predict only a few hundred signal events with significant background even with $3000 \mathrm{fb}^{-1}$ of data. With such small numbers of events, the detector must perform well and with very high efficiency; it must be even better than the present system to cope with SLHC particle fluxes and event pileup. If no Higgs exists, then deeper investigations of rarer channels will be required, in the presence of significant backgrounds which must be as well separated from signals as possible. Since the coming years may well provide unexpected discoveries, a new detector should also offer flexibility to adapt to new circumstances and physics opportunities.

\section{Present Tracker}

The CMS inner tracking system is designed to provide precise, efficient measurement of charged particle trajectories as well as accurate reconstruction of secondary vertices. It surrounds the interaction point and has a length of $5.8 \mathrm{~m}$ and a diameter of $2.5 \mathrm{~m}$ enclosed in a homogeneous $4 \mathrm{~T}$ magnetic field provided by the CMS solenoid.

The Tracker comprises a pixel detector with three barrel layers at radii between $4.4 \mathrm{~cm}$ and $10.2 \mathrm{~cm}$ and a silicon microstrip tracker with 10 barrel layers extending outwards to a radius of $1.1 \mathrm{~m}$. Each system is completed by endcaps which consist of two disks in the pixel detector and nine disks in the strip tracker on each side of the barrel, extending the acceptance of the tracker 
up to a pseudo-rapidity of $|\eta|<2.5$, including inner barrel disks. With about $200 \mathrm{~m}^{2}$ of active silicon area the CMS tracker is the largest silicon tracker ever built [5].

The tracker construction of 1,440 pixel and 15,148 strip detector modules required production methods and quality control procedures new to particle physics. A collaboration of 51 institutes with almost 500 physicists and engineers required about 15 years to design, develop and build this unique device, which was operated on the surface prior to installation in CMS, taking cosmic ray data in Spring 2007. It was inserted inside CMS in late December 2007 and connected to services by March 2008.

The pixel detector was inserted in July 2008. A key feature of this system is that it is quickly removable, because it was foreseen that beam-pipe bake-outs could be necessary between LHC operational periods. The first installation and connection required only a few days, as expected, and this is seen to be a major advantage for a future upgrade. A replacement of at least the inner layer was foreseen after several years of high luminosity operation as the sensors reach their radiation limits.

\section{A better tracker for SLHC?}

At present, there seems to be no physics justification to improve spatial and momentum measurement precision but tracking and vertexing performance must be maintained in the congested SLHC environment. Simulations of heavy ion events [6], where the track density is similar to SLHC, in the present tracker are encouraging. Inefficiencies arise from features of the design which can be improved; in particular an extra pixel layer would restore losses in track seeding. A new layout will be optimised for CPU-effective track finding in the p-p environment, for example to ensure jet reconstruction efficient remains high. The granularity must increase anyway because of leakage current noise after irradiation as well as track hit recognition.

The present system performance looks very promising and awaits collisions to be fully validated. The main criticism is that the material budget is larger than the earliest designs had anticipated. As with all tracking systems, multiple scattering, internal photon conversions, bremsstrahlung and hadronic interactions are undesirable, which can only be minimized by limiting material within the system. However, power required for on-detector electronics must be brought in by resistive cables and heat removed. Cooling, mechanical support and cables are inevitable, and grow in proportion to the power budget. To reduce this to the minimum was, and will continue to be, one of the largest challenges in the design.

One of the most difficult tasks to complete in the present Tracker, which imposes major constraints on an upgraded system, was installation of services. The cooling pipes, power cables and optical fibres follow complex, congested routes. The heat load of cables must be removed and this increases as the square of the current. Cable voltage drops already exceed ASIC supply voltages which have limited tolerance to over-voltage excursions and, in a system with no internal voltage regulation, this requires careful attention to power supplies and protection systems. In view of the major effort and substantial time which would be required and implications for other CMS sub-detectors whose services share the same volume, it is unlikely that the cables and cooling can be replaced for an SLHC Tracker. 


\section{Tracker input to Level 1 trigger}

This is foreseen to be required simply because single $\mu$, electron and jet trigger rates at Level 1 will greatly exceed $100 \mathrm{kHz}$ and cannot be reduced sufficiently by reasonable $\mathrm{p}_{\mathrm{T}}$ thresholds (figs $1 \& 2$ ). For muons, combinatorial background is mitigated in the High Level Trigger (HLT) by introducing tracking information [7,8]. In the case of the electron trigger, calorimeter isolation criteria alone do not provide sufficient rejection and association with pixel hits in the HLT provides the extra information needed to select electrons, demonstrating the candidate did not originate from a conversion or bremsstrahlung.

Simulations have not yet been completed under SLHC conditions but linear extrapolations from lower luminosity can be made, although likely to be optimistic, and demonstrate the expected rates. The present HLT shows that associating tracker data should improve muon momentum measurement and elimination of fake candidates (fig.1); a tracker hit in a limited $\eta$ - $\phi$ window should discriminate between ambiguous muon candidates and improve $\mathrm{p}_{\mathrm{T}}$. Because of the beam constraint, there will be little benefit from using a point close to the beam so the radial location can be in an outer layer.
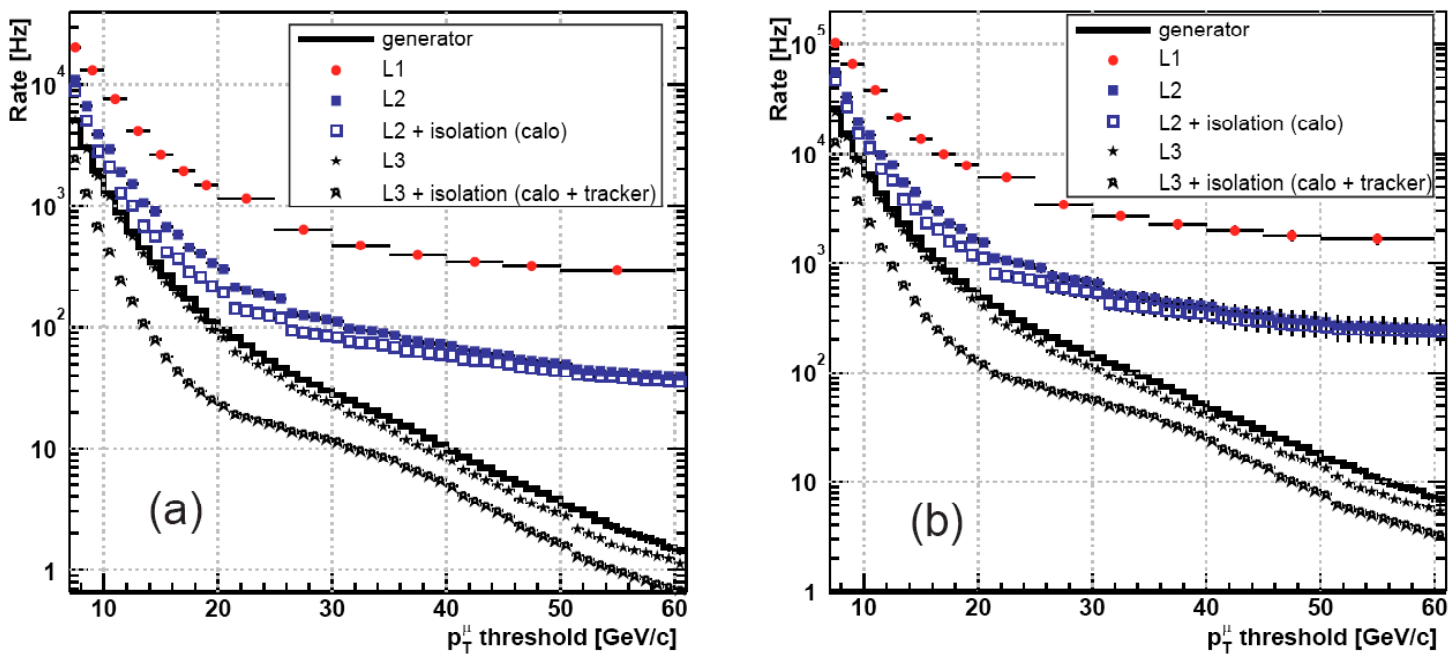

Fig. 1. The HLT single muon rates vs $p_{T}$ threshold for (a) low $\left(2 \times 10^{33} \mathrm{~cm}^{-2} \cdot \mathrm{s}^{-1}\right)$ and (b) maximum LHC $\left(10^{34} \mathrm{~cm}^{-2} \mathrm{~s}^{-1}\right)$ luminosity [7]. The rates are shown for Level 1 and at various HLT levels. Tracker information is included in Level 3.

The electron case is more subtle, as presently the HLT extrapolates an energetic calorimeter shower back to the pixel layers to search for an isolated hit (fig.3). Because of the high occupancy this is unlikely to work well at SLHC so alternatives are under study, possibly using coarse pixels in the inner region of the outer tracker. Little work has yet been done on jets but it is plausible that information on the proximity or local density of high $\mathrm{p}_{\mathrm{T}}$ hits would be useful.

The major difficult in easily implementing tracking triggers at Level 1 is that it will be impossible to transfer all hits off-detector for the decision logic. On-detector data reduction is therefore essential, since selective readout guided by another sub-detector also presents practical difficulties in providing a sufficiently fast intermediate level trigger. It is vital that delivering trigger functions must not degrade tracking performance by introducing more material. Trigger 
layers, if pixellated, are likely to be more power hungry than ordinary measuring layers, therefore there is a risk to the material budget.
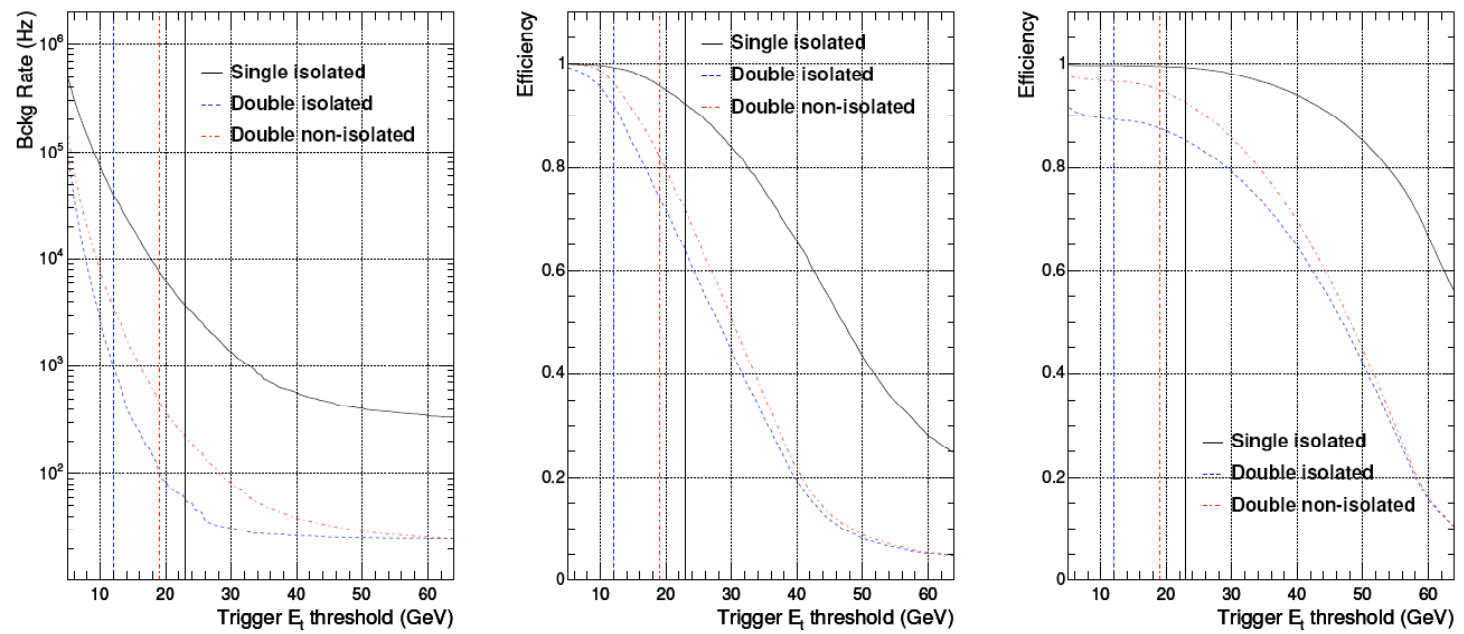

Fig 2. The rates for electromagnetic triggers as a function of threshold for various types of event (left: jet events, middle: $H->4$, right $H->\gamma \gamma)$ at a luminosity of $2 \times 10^{33} \mathrm{~cm}^{-2} . \mathrm{s}^{-1}[8]$. Scaling to $10^{35} \mathrm{~cm}^{-2} \cdot \mathrm{s}^{-1}$, it is clear that typical single isolated electron rates would be far higher than tolerable for practical thresholds.
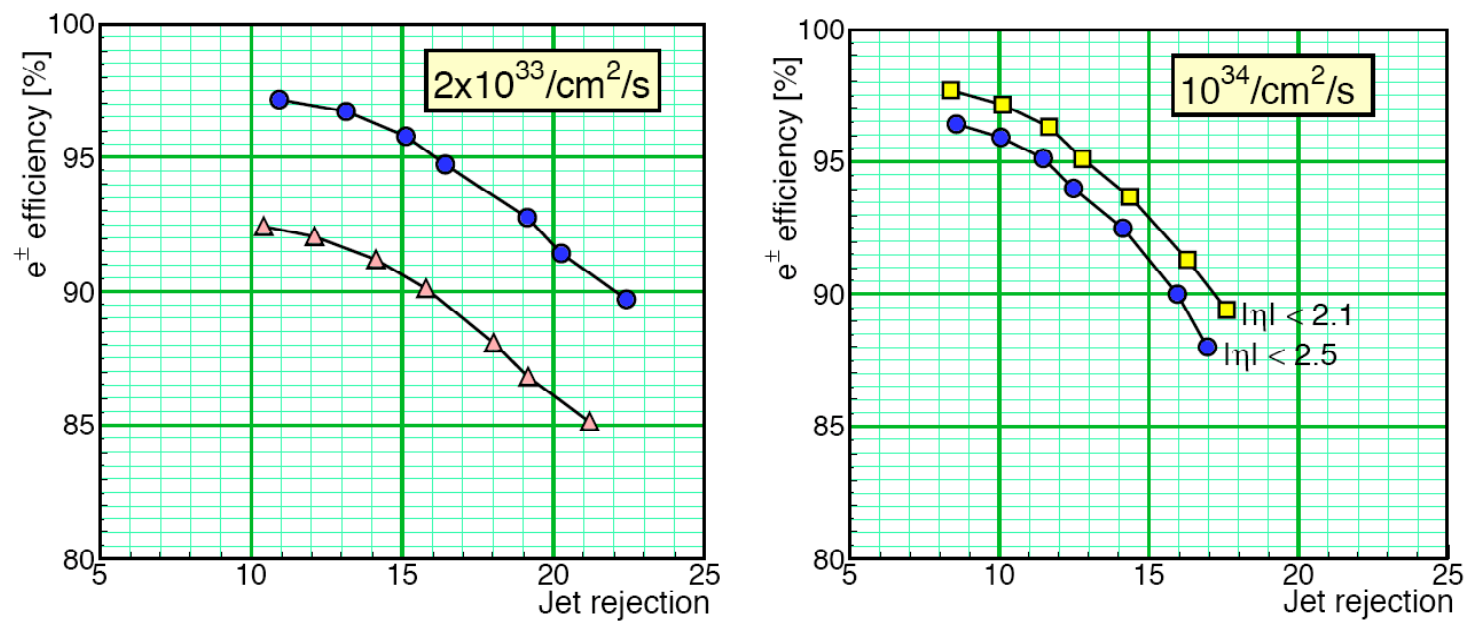

Fig. 3. Rejection vs efficiency for electron identification obtained from the HLT pixel matching and low (left) and maximum LHC (right) luminosity [8]. The upper curve shows the performance with the existing pixel detector.

One proposal would use cluster width information to eliminate low $\mathrm{p}_{\mathrm{T}}$ tracks $[9,10]$; this could require sensors to be sufficiently thick or have relatively fine pitch. It also appears problematic in the very forward region but could be effective to $|\eta|=1.7$.

An alternative $[11,12]$ would deploy closely spaced layers and compare the pattern of hits in contiguous sensor elements. Since the $\mathrm{p}_{\mathrm{T}}$ cut is set by the angle of a track in the layer, only nearby hits qualify high momentum candidates and the logic might be simple. Simulations support the basic concept but with unrealistically small elements for a practical detector so further investigations are needed, modelling a detector in more detail, as well as serious evaluations of power and speed issues. 


\section{Planning an Upgrade Project}

The SLHC planning assumption is that the first phase of the LHC machine upgrade might take place about five years after LHC start-up, with a long shutdown to reach $10^{35} \mathrm{~cm}^{-2} \mathrm{~s}^{-1}$ a few years later, including incremental improvements meanwhile to the machine infrastructure to ensure high reliability operation[13]. Based on previous experience, developing and building a new tracker of this size requires at least ten years, perhaps distributed as five years $R \& D$, two years qualification of components (and revisions, if necessary) followed by three years construction and assembly, with a final months for installation and to be ready for commissioning.

Many of those involved in building present tracking detectors consider such a schedule to be ambitious, especially given the new technical hurdles to be surmounted. What was learned from the past is that system design and attention to quality assurance are important considerations from a very early stage, and that cost was a significant driver for LHC detector designs from day one. These will certainly ensure plenty of hard work for the next generation of detector builders.

The CMS Tracker has begun with an organisation of Working Groups focussed on principal R\&D areas, addressing most of the items mentioned earlier [14]. Among the most important activities has been to develop simulations of a new system operating at SLHC luminosity [15]. The new CMS layout is still under discussion but is expected to start from with four barrel layers of pixels and expanded endcap system. Further out in radius, doublet layers to contribute to the trigger are under study, combined with short strips. The goal is to perform simulations and performance studies: by modelling the physical geometry, including numbers and location of layers, amount of material, "granularity" (e.g. pixels, mini-strips, size and thickness) as well as major electronic features, and potential trigger strategy, technology, and trigger algorithms.

Most of the software developers' attention until recently has been necessarily focussed on producing a common set of software tools to assist these studies. Naturally the existing software has been designed for LHC conditions, also emphasising early operation. Therefore it is no surprise that parts of the code are "hard wired" for the existing detector, and the code was not well optimised for extreme high pile-up or enormous numbers of tracks to be processed. However, flexible "straw-man" layouts have now been built which are starting to produce results and beginning to allow comparisons between different tracking system strategies and designs with different geometries.

\section{Power delivery}

Perhaps the most crucial technical question is how to deliver the required current to the new tracker. The present design uses over $15,000 \mathrm{~A}$ with internal power dissipation of $\sim 35 \mathrm{~kW}$. Even with advanced CMOS technologies, this is expected to increase and sensor leakage currents will also make significantly larger contributions to the total power budget at SLHC.

A key question is to calculate the power requirements, which at LHC are dominated by the front-end readout chip. For the future, analogue stages can be simulated but informed guesses 
are needed to estimate digital power, given uncertainties about such important details as functionality, readout architecture and Single Event Error mitigation. It appears possible to set a target of $500 \mu \mathrm{W} /$ channel for strips of a few cm length using a 130nm CMOS process [16], which is about a factor 5 reduction compared to the present. It also seems to be in agreement with similar studies for ATLAS [17]. The trigger requirements are more difficult to estimate and depend crucially on the architecture and granularity of dedicated layers, as well as data traffic and transfer to the off-detector processors. An estimate of $300 \mu \mathrm{W} /$ channel for $2.5 \mathrm{~mm} \times 100 \mu \mathrm{m}$ pixels is a plausible, but uncertain, extrapolation from existing components, which means that doublet layers dedicated to triggers would be vastly more demanding than other layers, with power density of $\sim 1.2 \mathrm{~kW} / \mathrm{m}^{2}$, compared to $\sim 120 \mathrm{~W} / \mathrm{m}^{2}$ for short strip layers.

On top of this must be added the power required for data transmission and buffering. Recent prototypes using a low current differential scheme [18] have demonstrated less than 10 $\mathrm{pJ} / \mathrm{bit}$ per link at $160 \mathrm{Mbit} / \mathrm{s}$ over $2 \mathrm{~m}$ using a low mass twisted pair cable with no crosstalk problems, which could be interesting for short distances. High speed bidirectional links using optical transmission over longer distances to off-detector processors currently estimate $\sim 400$ $\mathrm{pJ} / \mathrm{bit}$ [19], but may not yet be optimised for tracker applications.

Although estimates of power are still imprecise, overall requirements can be estimated. If possible, sensor power should be reduced using thin sensors, which will gain important benefits in the outer layers. Finer granularity should allow adequate noise performance but the channel count must still be constrained to the minimum compatible with adequate tracking performance. Even with a total readout power of $\sim 25-35 \mathrm{~kW}$ larger currents will be required. Since this is impossible using existing cables, which cannot be supplemented, radical solutions are required. The two alternative schemes under consideration are serial powering and on-detector DC-DC conversion, with custom circuits. Neither are proven or have been used in past systems and many problems remain to be solved. This area must develop significantly in the coming few years.

\section{Conclusions}

CMS is proceeding systematically to develop a new Tracker design using simulations to define the new layout. The performance of the present detector looks excellent, but in future it is essential to achieve similar performance and desirable to reduce the material budget. The largest challenges are power delivery and distribution and provision of triggering data at Level 1, but many other aspects of the new system are even more challenging than for the present detector.

\section{Acknowledgements}

I would like to pay tribute to my many colleagues in CMS who have contributed to the present, outstanding tracking detector which has now been completed and thank those whose contributions to the preparations to improve it for an even more demanding future are so inadequately acknowledged here. 


\section{References}

[1] F. Gianotti, M. Mangano, T. Virdee, et al., Physics Potential and Experimental Challenges of the LHC Luminosity Upgrade Eur. Phys. J. C39 (2004) 293-333. [doi:10.1140/epjc/s2004-02061-6.]

[2] M. Moll et al. (RD50 Collaboration) Development of radiation tolerant semiconductor detectors for the Super-LHC Nucl Instr and Meths A (2005) 546, 99-107

[3] The CMS Collaboration. CMS Expression of Interest in the SLHC. CERN/LHCC 2007-014 LHCCG-131 15 March 2007

[4] The CMS Collaboration. CMS Technical Design Report, Volume II: Physics Performance. J. Phys. G: Nucl. Part. Phys. 34(2007) [doi: 10.1088/0954-3899/34/6/E01]

[5] The CMS Collaboration The CMS experiment at the CERN LHC 2008 JINST 3 S08004 [doi: $10.1088 / 1748-0221 / 3 / 08 / \mathrm{S} 08004]$

[6] C Roland Track Reconstruction in Heavy Ion Events using the CMS Tracker Nucl. Instr. and Meths. A: 566, 1 (2006) 123-126

[7] The CMS Collaboration. CMS Data Acquisition and High-Level Trigger Design Report (2002) CERN/LHCC 02-26

[8] The CMS Collaboration. CMS Technical Design Report, Volume I: Detector Performance and Software (2006) CERN/LHCC 2006-001

[9] F Palla Proposal for a First Level Trigger using pixel detector for CMS at Super-LHC JINST 2 P02002 (2007) [doi: 10.1088/1748-0221/2/02/P02002]

[10] F. Palla and G. Parrini, Tracking in the trigger: from the CDF experience to CMS upgrade, PoS (Vertex 2007) 03

[11] C. Foudas, A. Rose, J. Jones, G. Hall. A Study of a Tracking Trigger at First Level for CMS at SLHC Proc. LECC 2005 Workshop, CERN Report CERN-2005-011(2005) 448-452

[12] J. Jones, A. Rose et al., Stacked Tracking for CMS at Super-LHC Proc. LECC 2006 Workshop (2007) CERN-2007-001, 130-134

[13] E. Shaposhnikova Intensity upgrade plans for CERN-LHC injectors CERN-AB-2008-065 (2008)

[14] Tracker Upgrade web pages http://cmsdoc.cern.ch/Tracker/Tracker2005/TKSLHC/index.html

[15] Tracker Upgrade Wiki pages https://twiki.cern.ch/twiki/bin/view/CMS/SLHCTrackerWikiHome

[16] M. Raymond, G. Hall CMS Microstrip Tracker Readout at the SLHC. Paper presented at TWEPP08 Topical Workshop on Electronics for Particle Physics, Sept 2008.

http://indico.cern.ch/conferenceDisplay.py?confId=21985

[17] J. Kaplon et al. The ABCN front end chip for ATLAS Inner Detector Upgrade, Paper presented at TWEPP-08 Topical Workshop on Electronics for Particle Physics, Sept 2008. $\underline{\mathrm{http}}$ ///indico.cern.ch/conferenceDisplay.py?confId=21985

[18] B Meier Design Studies of a Low Power Serial Data Link for a possible Upgrade of the CMS Pixel Detector Paper presented at TWEPP-08 Topical Workshop on Electronics for Particle Physics, Sept 2008. http://indico.cern.ch/conferenceDisplay.py?confId=21985

[19] P Moreira The GBT Bi-directional links and Data system in Proceedings of LECC 2006, 12th Workshop on Electronics for LHC and Future Experiments (2007) CERN-2007-001, 93-96. 